\title{
Non-Associative Learning in Larval Zebrafish
}

\author{
Jonathan D Best*,', Stéphane Berghmans', Julia JFG Hunt', Samantha C Clarke', Angeleen Fleming', \\ Paul Goldsmith' and Alan G Roach' \\ 'DanioLabs Ltd (a wholly owned subsidiary of VASTox plc), Unit 7330, Cambridge Research Park, Cambridge, UK
}

\begin{abstract}
Habituation, where a response is reduced when exposed to a continuous stimulus is one of the simplest forms of non-associative learning and has been shown in a number of organisms from sea slugs to rodents. However, very little has been reported in the zebrafish, a model that is gaining popularity for high-throughput compound screens. Furthermore, since most of the studies involving learning and memory in zebrafish have been conducted in adults, we sought to determine if zebrafish larvae could display non-associative learning and whether it could be modulated by compounds identified in previous rodent studies. We demonstrated that zebrafish larvae (7 days post fertilization) exhibit iterative reduction in a startle response to a series of acoustic stimuli. Furthermore, this reduction satisfied criteria for habituation: spontaneous recovery, more rapid reductions in startle to shorter intertrial intervals and dishabituation. We then investigated the pathways mediating this behavior using established compounds in learning and memory. Administration of rolipram (PDE4 inhibitor), donepezil (acetylcholinesterase inhibitor), and memantine ( $N$-methyl-D-aspartic acid (NMDA) receptor antagonist) all increased the acoustic startle response and decreased habituation in the larvae, similar to previous rodent studies. Further studies demonstrated that NMDA blocked the memantine response and the effect of donepezil was blocked by mecamylamine but not atropine suggesting that the donepezil response was mediated by nicotinic rather than muscarinic receptors. Zebrafish larvae possess numerous advantages for medium to high-throughput screening; the model described herein therefore offers the potential to screen for additional compounds for further study on cognition function.

Neuropsychopharmacology (2008) 33, 1206-1215; doi:I0.1038/sj.npp.130 I489; published online 20 June 2007
\end{abstract}

Keywords: zebrafish; non-associative learning; cholinergic; rolipram; cognition; NMDA

\section{INTRODUCTION}

Habituation is one of the simplest forms of learning, resulting in the reduction of a response to a repeated stimulus (Thompson and Spencer, 1966). The reduction in the response can last from minutes to hours depending on the type of stimulus and study conducted. This type of response, often referred to as non-associative learning, has been shown by a variety of organisms from the invertebrates Aplysia, Tritonia, and Drosophila, to rats, using different types of stimuli (Marcus et al, 1988; Pletnikov et al, 1995; Brown, 1998; Cho et al, 2004). One of the prominent behaviors studied in vertebrates is the startle response, a fast contraction of body muscles caused by a sudden acoustic, tactile, or visual stimulus mediated by simple neuronal circuitry (Koch, 1999). It is interpreted to be an evolutionarily conserved form of protection against potential danger and predators. Increase of the acoustically generated startle (commonly called the acoustic startle response; ASR) is known to have different causes such as

*Correspondence: Dr JD Best, DanioLabs Ltd (a wholly owned subsidiary of VASTox plc), Unit 7330, Cambridge Research Park, Beach Road, Waterbeach, Cambridge CB25 9TN, UK, Tel: + 441223 706460, Fax: + 44 1223 70646I, E-mail: jonathan.best@daniolabs.com Received 29 March 2007; revised 17 May 2007; accepted 23 May 2007 sensitization, fear-potentiation, or certain drugs, whereas decrease of the ASR can be caused by habituation, pre-pulse inhibition, or certain drugs (Koch, 1999). Previous studies in rodents have demonstrated an enhancing effect on the ASR of compounds affecting the phosphodiesterase and $N$-methyl-D-aspartic acid (NMDA) pathways (Kehne et al, 1991; Klamer et al, 2004). Recent research studies suggest that zebrafish have a rich and complex sense of hearing, sharing many anatomical features of the inner ear with other vertebrates, including humans (Whitfield, 2002). By 5 days post fertilization (d.p.f.), free-swimming zebrafish larvae have developed morphological structures for hearing that allow them to perform an ASR when exposed to acoustic stimuli (Bang et al, 2002; Zeddies and Fay, 2005). Since most compound screens are usually performed in rodents, the zebrafish may offer an alternative and unique approach to identifying novel therapeutics; the startle response being used as a measure of information processing and attentional deficits.

The zebrafish model system possesses numerous advantages for medium- to high-throughput screening. These include (i) the relative ease of maintaining large stocks of animals, (ii) its high fecundity, providing large numbers of animals for analysis, and (iii) rapid embryonic development ex utero, which facilitates experimental manipulation and allows the direct observation of tissue formation and 
organogenesis in vivo (Westerfield, 2000). The organization of the genome and the genetic pathways controlling signal transduction and development are highly conserved between zebrafish and man (Postlethwait et al, 2000). These properties have established the zebrafish as an excellent model system that is relevant to studies of human diseases (Grunwald and Eisen, 2002). Zebrafish have also become the focus of neurobehavioral studies since larvae display learning, memory, sleep, drug addiction, and behavior phenotypes that are quantifiable and related to those seen in man (Zhdanova et al, 2001; Cahill, 2002; Guo, 2004; Orger et al, 2004; Ninkovic et al, 2006).

In terms of the neuroanatomy, despite some notable differences between teleost and mammals such as the layout of the forebrain (reviewed by Wullimann and Mueller, 2004) and the structure and function of the optic tectum (Luque et al, 2005), the overall organization of the zebrafish brain is similar to other vertebrates, even encompassing structures of the lateral pallium which appear to be homologous to the mammalian hippocampus, lending support for the application of zebrafish as a useful model to evaluate vertebrate behavior (Tropepe and Sive, 2003).

In addition, since the larvae can live in as little as $200 \mu \mathrm{l}$ of fluid, only micrograms of compound are needed for screening. Thus, in vivo analysis of the effects of compounds can be undertaken at much earlier stages in drug development with a higher throughput, a process facilitated by the fact that zebrafish are DMSO tolerant and readily absorb compounds from the water (Goldsmith, 2004).

The study described herein set out to determine whether zebrafish habituate to acoustic stimuli and whether this simple form of non-associative learning could be modulated by compounds affecting pathways similar to those identified in previous rodent studies.

\section{MATERIALS AND METHODS}

\section{Animals}

All procedures were conducted in accordance with the Animals (Scientific Procedures) Act of 1986 (UK) and its associated guidelines. Fish were reared under standard conditions (Haffter et al, 1996; Westerfield, 2000). Embryos were collected from natural spawnings of group breedings and staged according to established criteria (Kimmel et al, $1995)$ and reared in embryo medium $(5 \mathrm{mM} \mathrm{NaCl}, 0.17 \mathrm{mM}$ $\mathrm{KCl}, 0.33 \mathrm{mM} \mathrm{CaCl}_{2}, 0.33 \mathrm{mM} \mathrm{MgSO}_{4}, 10^{-5} \%$ methylene blue $\mathrm{pH} 7.2$ ) in the dark at $28.5^{\circ} \mathrm{C}$. Wild-type zebrafish were descendants of the $\mathrm{AB}$ or WIK strain obtained from Sheffield University (UK).

\section{ASR Assay}

Zebrafish larvae were placed in a 96-well mesh plate (Millipore, Watford, UK) within a teardrop tray containing $200 \mu \mathrm{l}$ of E3 embryo medium, one fish per well. Since zebrafish larvae do not show responses to acoustic stimuli until 5 d.p.f., 6-9 d.p.f. larvae were used for behavioral testing. For pharmacological assessment of the ASR, 6 d.p.f. larvae were exposed to compounds added to the E3 medium. Twenty-four hours later compound solutions were replaced (larvae transferred into a new teardrop tray containing fresh compound solution), after which they were placed in the tracking system. An automated live tracking system comprising of a Sony XC EI50 CE Camera (Tracksys Ltd, Nottingham, UK) and Ethovision software (Noldus, Wageningen, The Netherlands) was used to monitor the larvae. Fifteen minutes later the larvae were exposed to a sequence of auditory tones synchronized by the Ethovision software.

\section{Induction of Habituation and Dishabituation of the ASR}

Auditory cues of $200 \mathrm{~Hz}$ in frequency, $113 \mathrm{~dB}$ measured using NM102 Noise Meter (NoiseMeter Ltd, Burton Fleming, UK) placed above the microwell plate and $0.6 \mathrm{~s}$ in length were produced from side-mounted speakers (Bell Packard; placed $10 \mathrm{~cm}$ away from the side of the 96-well plate) connected to a Dell computer and given at 1, 5, or $20 \mathrm{~s}$ intervals (referred to as the intertrial interval; ITI). An auditory tone session consisted of up to 100 tones, with up to four sessions being given with 15 min recovery period between each episode of auditory stimulation. The ASR was analyzed for each individual larva by measuring the distance moved in response to each auditory stimulus; this provided a quantitative readout of the startle response. The distance moved was defined as the distance moved by the larvae for $1 \mathrm{~s}$ from the beginning of the auditory stimulus. In the dishabituation experiment, a $2 \mathrm{~s}$ light pulse (white LED light: $560 \operatorname{lux}, 465-700 \mathrm{~nm}$ ) was also given after the 80th tone. The distance moved $(\mathrm{cm})$ by each larva over the $1 \mathrm{~s}$ period from the start of each individual auditory stimulus was computed by the software.

\section{Statistical Analysis}

All analysis was conducted using Prism 4.03 (GraphPad Software Inc., San Diego, CA). The mean and standard errors of the mean of the distances moved by the larvae in individual treatment groups were calculated. Group data were analyzed using Student's $t$-tests or the appropriate one-way or two-way ANOVA and post hoc $t$-tests. The area under the curve (AUC) was calculated using $Y=0$ and peaks less than $10 \%$ of the distance from minimum to maximum $Y$ were ignored.

\section{Compound Administration and Toxicity Evaluation}

Donepezil hydrochloride (Sequoia Research Products Ltd, Pangbourne, UK), memantine hydrochloride (Kemprotec Ltd, Middlesbrough, UK), rolipram, NMDA, mecamylamine hydrochloride, and atropine sulfate (Sigma-Aldrich, Poole, UK) were solubilized in DMSO and diluted in E3 medium to give the requisite test concentration. All solutions were diluted such that the final DMSO concentration in the wells was $1 \%$. Equivalent DMSO vehicle controls were evaluated concurrently with the test compounds. Groups of between 24 and 48 larvae underwent behavioral testing. The compound treatment groups consisted of 12 larvae and included a DMSO vehicle-treated control group. Compounds were initially evaluated for toxicity before testing in the ASR assay. Briefly, toxicity was ascertained in 7 d.p.f. zebrafish larvae after $24 \mathrm{~h}$ exposure and was established 

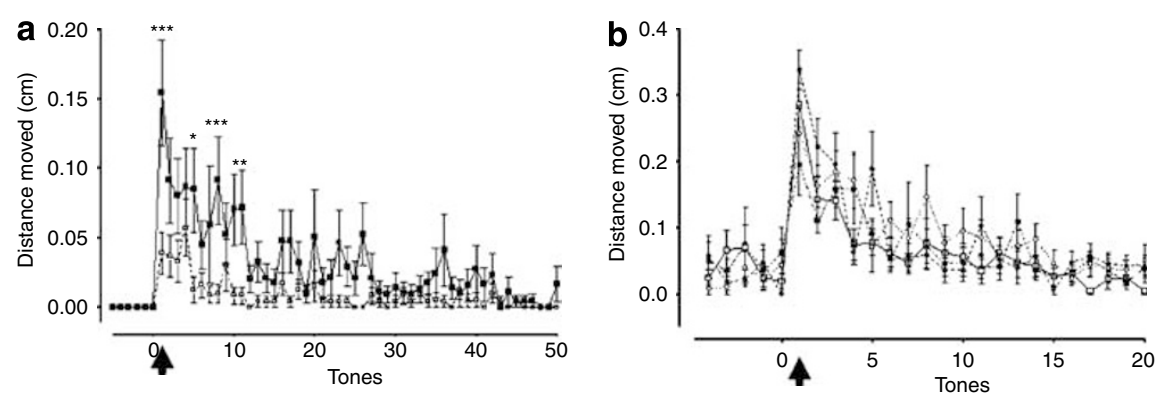

Figure I Sequential auditory cues cause iterative reduction in startle response in zebrafish. Effect of strain and age on startle response. Graphs of distance moved against auditory stimuli for (a) AB ( $\square$ dotted line) vs WIK ( $\square$ solid line) strains, 7 d.p.f. and (b) different ages of WIK strain (6 d.p.f., $\square$ dotted line; 7 d.p.f., $\square$ solid line; 8 d.p.f., $\bigcirc$ dotted line; 9 d.p.f, $\bigcirc$ dotted line). Data points represent average distance moved \pm SEM $(n=24 /$ data point). $* * * *<<0.000$, *** $p<0.001$, $* p<0.05$. Arrow denotes beginning of auditory stimuli.

either by death or a reduced capacity of the fish to startle to a physical tap applied to the dish. Any larvae displaying a reduced tap startle were evaluated under a microscope (Zeiss Stemi-2000C) and gross morphology, cardiovascular development, and signs of edema were scored to determine the extent of the compound effect. Donepezil, rolipram, and memantine were tested initially at 3,10 , and $30 \mu \mathrm{M}$ in the ASR assay. The other compounds were assessed for toxicity and the highest concentration, which did not induce toxicity, was used in subsequent assays. The 6 d.p.f. larvae were placed in the solution containing the compound for $24 \mathrm{~h}$ after which time the compounds were refreshed and live video tracking of the 7 d.p.f. zebrafish larvae was performed immediately and analyzed as described above.

\section{RESULTS}

\section{Comparison of Strain and Age}

A number of different volumes $(76,89,108,113 \mathrm{~dB})$ and frequencies $(50,100,200,400,600,800,1000 \mathrm{~Hz})$ were initially investigated to determine which combination gave the best response (data not shown), and it was found that $200 \mathrm{~Hz}$ at the top volume of $113 \mathrm{~dB}$ gave the greatest and most consistent startle response. To determine which strain of zebrafish would be optimal for use in the ASR assay, the widely used $A B$ and WIK strains were compared. Twentyfour fish at 7 d.p.f. of each strain were exposed to 50 auditory stimuli with $1 \mathrm{~s}$ ITI. There was an effect of the sound stimulus on the distance moved for both the $\mathrm{AB}$ and WIK strain (repeated measures one-way ANOVA, $\mathrm{F}=2.518, p<0.0001$, $A B ; F=8.761, p<0.0001$, WIK), with both strains significantly increasing the distance moved compared to the baseline recording (tone $0 v s$ tone $1, p<0.001$ for both strains) in keeping with an ASR (Figure 1a). The movement in response to the first auditory tone was always greater than the distance recorded to subsequent stimuli. Furthermore, both strains demonstrated iterative reductions of the distance moved, with the $A B$ strain returning to baseline by the fifth tone and the WIK strain consistently returning to baseline after the 12th tone. This effect was consistent with the learning phenomenon of habituation. When the two strains were compared by two-way ANOVA, there was a significant difference between the distances moved by the two strains $(\mathrm{F}=76.24)$; the WIK strain being found to move a four times greater distance to the first tone compared to the $A B$ strain $(p<0.001)$ and was significantly different at a number of time points following the first tone (Figure 1a).

Since the distance moved to the auditory cues was greater in the WIK strain, all further experiments were performed using this strain. Six to nine d.p.f. larvae were compared to determine the responses at various stages of development. Twenty-four fish per age group were exposed to 25 tones with $1 \mathrm{~s}$ ITI. The presentation of the auditory tones showed effects in larvae at all ages tested (Figure 1b, repeated measures one-way ANOVA; $\mathrm{F}=2.319, p<0.001,6$ d.p.f.; $\mathrm{F}=6.077$, $p<0.0001,7$ d.p.f.; $\mathrm{F}=9.170, p<0.0001,8$ d.p.f.; $\mathrm{F}=4.348$, $p<0.0001$, 9 d.p.f.); the distance moved increasing significantly in response to the first tone $(p<0.01)$ compared to the baseline in larvae at all ages. This increase in distance moved reduced to baseline by the sixth tone, consistent with habituation. When the effect of the tones was compared across the groups by two-way ANOVA, there was no significant difference between the different ages $(\mathrm{F}=1.873$, NS).

\section{Determination of Habituation}

To investigate whether the iterative reductions seen in response to the sequential tones were a result of habituation, a number of studies were conducted. To determine if the fish were able to spontaneously recover, two series of auditory tones separated by $15 \mathrm{~min}$ were administered to twenty-four 7 d.p.f. WIK larvae. There was an effect of the first set of tones (Figure 2a, repeated measures one-way ANOVA, $\mathrm{F}=4.423, p<0.0001$ ), with the distance moved increasing significantly compared to the baseline movement $(p<0.001)$. The distance moved decreased with the sequential cues, returning to baseline levels by the fourth tone. The process was repeated $15 \mathrm{~min}$ later, and the fish responded to the second set of tones (repeated measures one-way ANOVA, $\mathrm{F}=3.030, p<0.0001$ ), with the first tone of the second session again significantly increasing the distance the fish moved $(p<0.001)$ demonstrating therefore that the fish had recovered between sessions. Interestingly, when the first and second set of tones were compared, there was a greater effect of the second trial on the distance moved (repeated measures two-way ANOVA, $\mathrm{F}=52.85$, $p<0.0001)$ : the distance moved to the fourth tone of the second series was significantly greater that the distance moved to the fourth tone of the first set of cues $(p<0.001)$, although the distance moved to the tones returned to baseline by the fifth tone. 

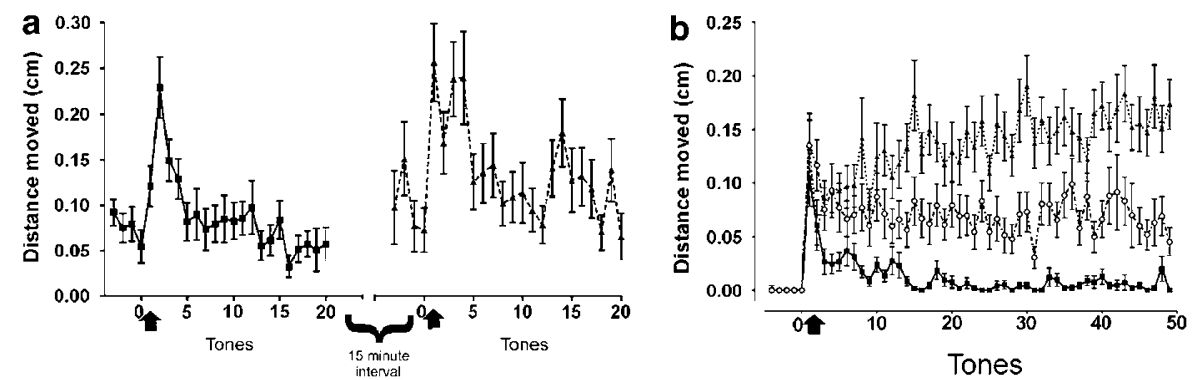

Figure 2 Spontaneous recovery from iterative reduction occurs after $15 \mathrm{~min}$ interval and iterative reduction tends to occur more quickly at shorter ITI. (a) Graph of distance moved against auditory stimuli for first set of 20 auditory tones ( solid line) separated by I 5 min of no noise (denoted by curly brackets) followed by a second set of 20 auditory tones ( $\boldsymbol{\Delta}$ dotted line). Data points represent average distance moved \pm SEM ( $n=24 /$ data point). Arrow denotes beginning of auditory stimuli. (b) Graph of distance moved against auditory stimuli for I s ITI ( $\mathbf{\square}$ solid line), $5 \mathrm{~s}$ ITI ( $\bigcirc$ dotted line), 20 s ITI ( $\mathbf{\Delta}$ dotted line). Data points represent average distance moved $\pm \operatorname{SEM}(n=48 /$ data point). Arrow denotes beginning of auditory stimuli.

Different ITIs were tested to determine whether the iterative reductions took place at different rates. Forty-eight fish per group were exposed to 50 tones at 1,5 , and $20 \mathrm{~s}$ intervals (Figure $2 \mathrm{~b}$ ). In all groups, the presentation of an auditory stimulus produced a significant movement effect (repeated measures one-way ANOVA; $\mathrm{F}=5.684, p<0.0001$, $1 \mathrm{~s}$ ITI; $\mathrm{F}=2.839, p<0.0001,5 \mathrm{~s}$ ITI; $\mathrm{F}=6.171, p<0.0001$, $20 \mathrm{~s}$ ITI). In all cases, the first tone caused a significant increase in the distance moved $(p<0.001)$; with $1 \mathrm{~s}$ ITI the distance moved decreased and returned to baseline as seen previously by the fifth and sixth tone, whereas the $5 \mathrm{~s}$ ITI decreased slightly compared to the movement to the initial tone but not to the same level as the pre-tone baseline. The $20 \mathrm{~s}$ ITI maintained the same level or slightly increased (although not significantly) the distance moved compared to the first tone.

\section{Determination of Dishabituation}

Dishabituation occurs where, following habituation to stimulus $A$, presentation of stimulus $B$ causes a reinduction of the response to stimulus A. The stimulus B in this study was a light pulse, which is known to cause a startle response in zebrafish (Easter and Nicola, 1996). Separate groups of forty-eight 7 d.p.f. zebrafish of the WIK strain were exposed between 80 and 100 auditory tones of $5 \mathrm{~s}$ ITI, with some groups experiencing a $2 \mathrm{~s}$ light pulse after the 80th tone (Figure 3). There was a significant effect of the acoustic stimuli $\quad(\mathrm{F}=2.401, \quad p<0.0001$, Figure $3 \mathrm{a} ; \mathrm{F}=3.330$, $p<0.0001$, Figure $3 \mathrm{~b} ; \mathrm{F}=4.685, p<0.0001$, Figure $3 \mathrm{c}$; $\mathrm{F}=3.191, p<0.0001$, Figure $3 \mathrm{~d}$ ), with the first auditory tone causing a significant increase in the distance moved $(p<0.001)$. As demonstrated previously, the distance moved decreased progressively returning to a level similar to the pre-tone baseline (Figure 3a-c). However, when a $2 \mathrm{~s}$ light pulse was added after the 80th tone, the 81st tone caused an increase in the distanced moved $(p<0.001)$ equivalent to the first tone $(p>0.05)$. Further combinations of tones and a light pulse demonstrated that this effect was consistent with dishabituation (Figure $3 c$ and $d$ ).

\section{Pharmacological Modulation of ASR}

A number of pharmacological agents are known to affect the ASR in rodents. We set out to determine if this behavior in zebrafish was affected in a similar manner to that seen in rodents. Groups of twelve 7 d.p.f. WIK zebrafish treated with $1 \%$ DMSO or test compound in $1 \%$ DMSO were exposed to 50 tones with $1 \mathrm{~s}$ ITI (Figures 4-7, for clarity only 20 tones are shown in the distance moved graphs).

\section{Phosphodiesterase IV Inhibition}

A previous study in the rat had demonstrated an enhanced startle response with the administration of rolipram (Kehne et al, 1991). Thus, we assessed the effects of rolipram on the ASR in the zebrafish larvae (Figure 4). There was an effect of the auditory tones in both the control group (Figure 4a, repeated measures one-way ANOVA, $\mathrm{F}=4.117, p<0.0001$ ) and the group treated with $3 \mu \mathrm{M}$ rolipram (Figure $4 \mathrm{a}$, repeated measures one-way ANOVA, $\mathrm{F}=3.737, p<0.0001$ ), with the first tone eliciting a significant increase in the distance moved compared to baseline $(p<0.001$, tone $0 v s$ tone 1 for both control and rolipram). Furthermore, both groups showed a return of the ASR to baseline after consecutive stimuli consistent with habituation. Comparison of the control group to the $3 \mu \mathrm{M}$ rolipram group indicated a significant effect of treatment with rolipram (two-way ANOVA; $\mathrm{F}=29.07, p<0.0001$ ); rolipram increasing the distance moved to the first tone by 2.5 times $(p<0.0001)$. Furthermore, in contrast to the controls, rolipram significantly increased the distance moved for the next six tones. An effect of treating the larvae with rolipram was also seen with 10 and $30 \mu \mathrm{M}$ (two-way ANOVA $v s$ control; Figure $4 \mathrm{~b} 10 \mu \mathrm{M}, \mathrm{F}=18.99$; Figure $4 \mathrm{c}$ $30 \mu \mathrm{M}, \mathrm{F}=19.38$; both $p<0.0001$ ) although analysis of the AUCs confirmed that $3 \mu \mathrm{M}$ gave the greatest response of $2-3$ times the control value (Figure $4 \mathrm{~d} ; p<0.05$ ).

\section{Cholinergic Pathways}

To determine if the ASR could be affected by cholinergic mechanisms, donepezil was also compared to its vehicle control at 3, 10, and $30 \mu \mathrm{M}$ (Figure 5). In independent experiments, both 3 and $10 \mu \mathrm{M}$ demonstrated an enhancing effect compared to their respective control groups (two-way ANOVA; Figure $5 \mathrm{a}, 3 \mu \mathrm{M}, \mathrm{F}=10.90, p<0.001$; Figure $5 \mathrm{~b}$, $10 \mu \mathrm{M}, \mathrm{F}=3.237, p<0.0001)$, with a significant increase in the distance moved occurring after the first tone $(p<0.001)$. However, treatment with donepezil at $30 \mu \mathrm{M}$ revealed a 

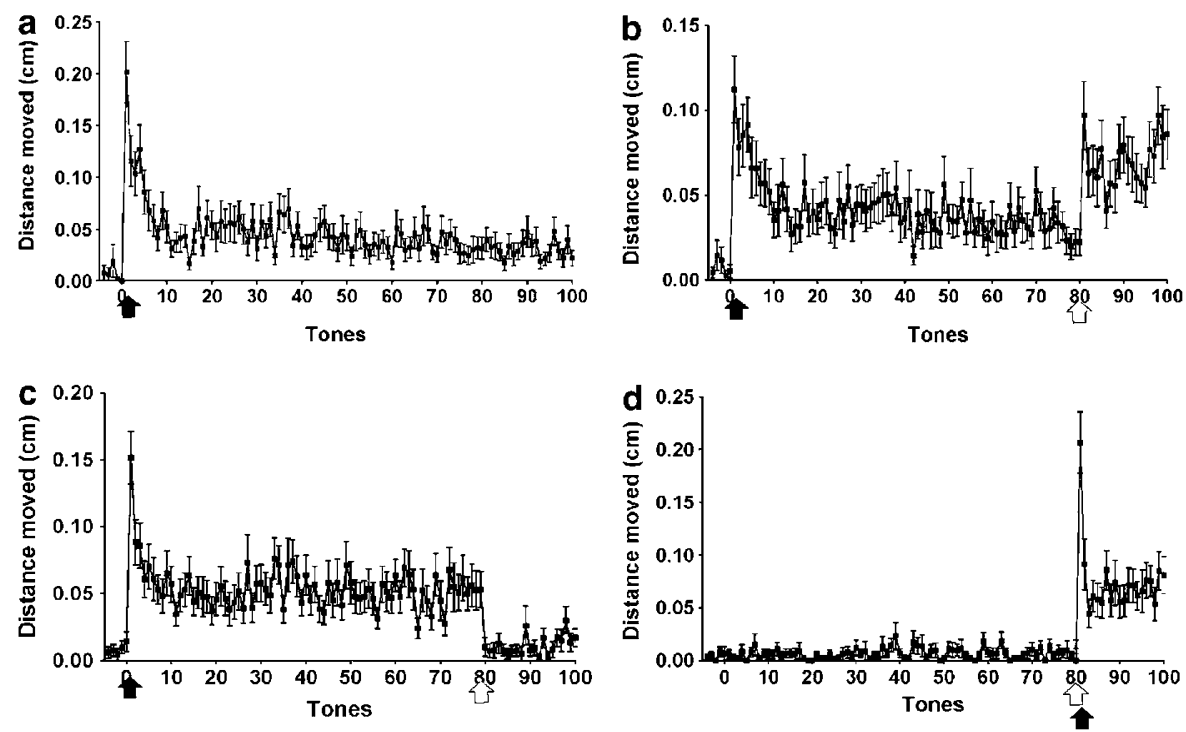

Figure 3 Reversal of iterative reduction when an alternate stimulus is presented between iterated trials (dishabituation). Graphs of distance moved against auditory stimuli ( solid line). (a) One hundred auditory stimuli, (b) 100 auditory tones with two second light pulse interposed between 80th and 81 st tone, (c) 80 tones with $2 \mathrm{~s}$ light pulse following 80th tone and no further auditory tones but movement recorded for equivalent of $8 \mathrm{Ist}$ to I00th tone, (d) no auditory tones given for the equivalent of 80 tones followed by a $2 \mathrm{~s}$ light pulse followed by first auditory tone at the equivalent time of the 80 th tone. Data points represent average distance moved \pm SEM ( $n=24 /$ data point). Black arrow denotes beginning of auditory stimuli; white arrow denotes $2 s$ light pulse.
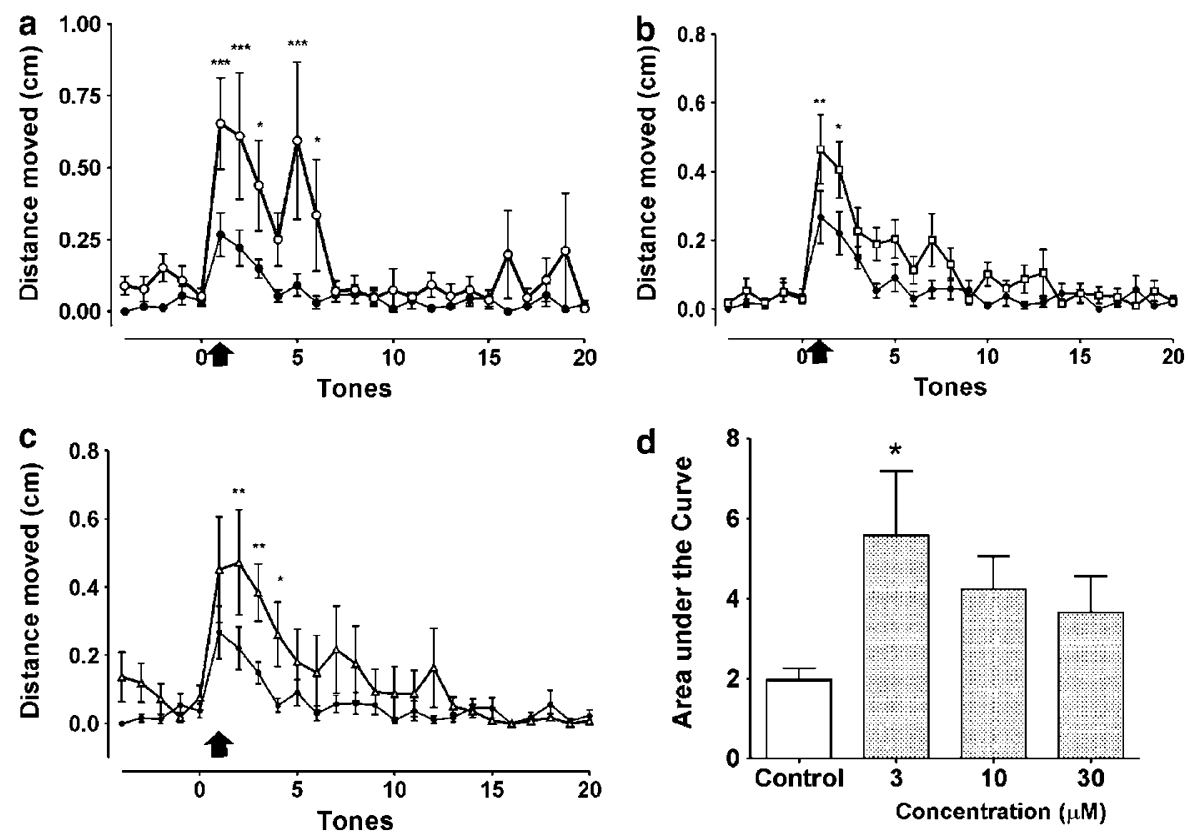

Figure 4 Rolipram enhances startle response in zebrafish larvae. Graphs of distance moved against auditory stimuli for (a) $3 \mu M$ ( $O$ thick solid line), (b) $10 \mu \mathrm{M}$ ( $\square$ thick solid line), and (c) $30 \mu \mathrm{M}$ rolipram ( $\triangle$ thick solid line) vs I\% DMSO control ( thin solid line). Data points represent average distance moved \pm SEM ( $n=12 /$ data point). $* * * *<0.0001$, $* * p<0.001$, $* p<0.05$ vs corresponding control value. Arrow denotes beginning of auditory stimuli. (d) Bar graph of AUC of graphs (a, b, and c) for control fish (clear) vs concentration response for rolipram (shaded). Bars represent the average AUC \pm SEM; $n=12$ per group. ${ }^{*} p<0.05$.

reduced startle to the dish tap (data not shown) and therefore was not tested further. When compared with the control group, $10 \mu \mathrm{M}$ donepezil gave a significant increase of 2.5 times the distance moved $(p<0.001)$ after the second tone. This potentiation of the ASR continued up to the 12th tone demonstrating a similar effect to that seen with rolipram. Again, the AUCs (Figure $5 \mathrm{c}, 3 \mu \mathrm{M}$; Figure 5d, $10 \mu \mathrm{M})$ confirmed that $10 \mu \mathrm{M}$ gave the greatest effect, with a fourfold increase compared to the control group (Figure $5 \mathrm{~d}$, Student's $t$-test, $p<0.0001)$. Thus, $10 \mu \mathrm{M}$ concentration of donepezil was used in subsequent studies.

To determine if the effect of donepezil on the ASR was mediated via a subset of cholinergic receptors, mecamylamine and atropine were tested in conjunction with donepezil. Initially, mecamylamine was combined with donepezil (Figure 6a and Supplementary Figure 1). Alone 

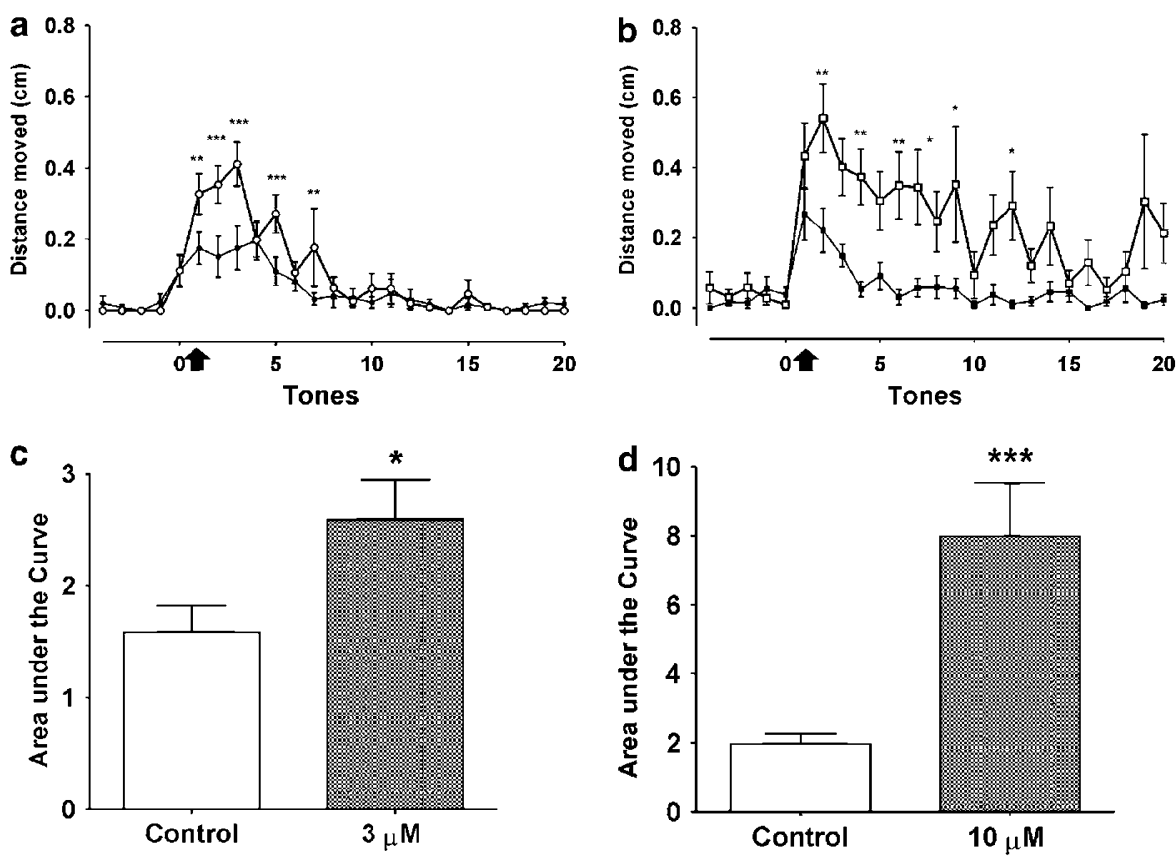

Figure 5 Donepezil enhances startle response in zebrafish larvae. Graphs of distance moved against auditory stimuli for (a) $3 \mu M$ donepezil ( $O$ thick solid line) vs I\% DMSO control ( thin solid line) and (b) $10 \mu \mathrm{M}$ donepezil ( $\square$ thick solid line) vs I\% DMSO control ( $\mathbf{\square}$ thin solid line). Data points represent average distance moved \pm SEM ( $n=12$ /data point). ***** $p<0.000$ I, *** $p<0.00$ I, $* p<0.05$ vs corresponding control value. Arrow denotes beginning of auditory stimuli. (c) Bar graph of AUC of graph A for control fish (clear) vs $3 \mu \mathrm{M}$ donepezil (shaded). (d) Bar graph of AUC of graph B for control fish (clear) vs $10 \mu \mathrm{M}$ donepezil (shaded). Bars represent the average AUC $\pm S E M ; n=12$ per group. $* p<0.05, * * * *<0.000 \mid$ vs control group.
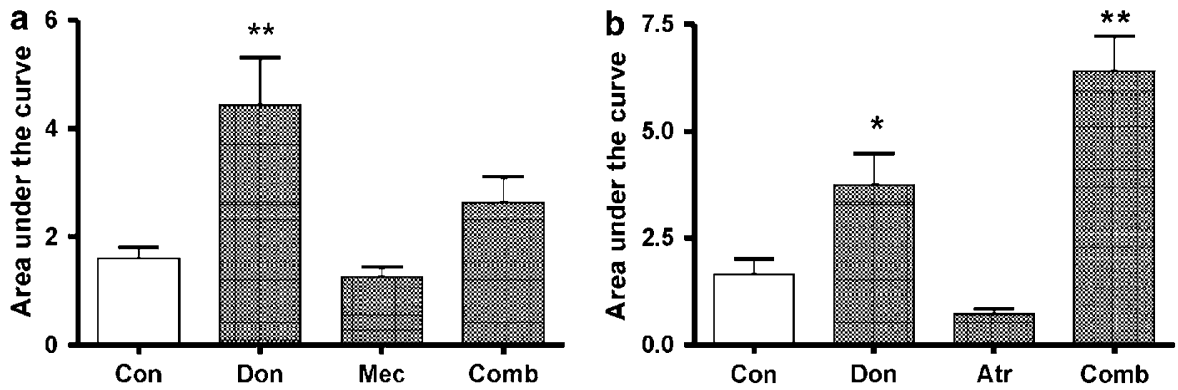

Figure 6 Mecamylamine but not atropine attenuates donepezil effect on startle response in zebrafish larvae. (a) Bar graph of AUC of control (Con) fish (clear) vs donepezil (Don), mecamylamine (Mec), and combination (Comb) (shaded). Bars represent the average AUC \pm SEM; $n=12$ per group. *** $<0.00$ I vs control group. (b) Bar graph of AUC of control (Con) fish (clear) vs donepezil (Don), atropine (Atr), and combination (Comb) (shaded). Bars represent the average AUC \pm SEM; $n=12$ per group. $* p<0.05$, $* * 00.001$ vs control group.

mecamylamine $(30 \mu \mathrm{M})$ did not have a significant effect on the habituation of the startle response compared to the control group (Mec, Figure 6a; Supplementary Figure 1A). However, when combined with donepezil, the increased response of the distance moved seen with donepezil alone (Don, Figure 6a; Supplementary Figure 1B, two-way ANOVA; $\mathrm{F}=38.24, p<0.0001)$ was abolished completely by $30 \mu \mathrm{M}$ mecamylamine (combination (Comb), Figure 6a; Supplementary Figure 1C).

Treatment with atropine $(100 \mu \mathrm{M})$ alone caused a significant increase in the habituation response (Supplementary Figure 2A, two-way ANOVA; $\mathrm{F}=17.66, p<0.0001$; atropine (Atr), Figure 6b), the startle response returning more rapidly to baseline after the third and fourth tone compared to the control, demonstrating that this concentration was effecting the larvae's behavior. However, when combined with donepezil, atropine did not abolish the increased response (Figure 6b, $p<0.05$ and $p<0.01$, donepezil (don) and combination (Comb), respectively; two-way ANOVA, Supplementary Figure $2 \mathrm{~B}, \mathrm{~F}=24.81$, $p<0.0001$; Supplementary Figure $2 \mathrm{C}, \mathrm{F}=137.6, p<0.0001$ ).

\section{NMDA Pathway}

In a separate experiment, larvae exposed to the NMDA receptor antagonist memantine were compared to control vehicle-treated fish (Figure 7a, Supplementary Figure 3). Overall, there was a significant increase on the ASR in both the groups treated with 10 and $30 \mu \mathrm{M}$ memantine compared to the control (Supplementary Figure 3B and C, two-way ANOVA, $\mathrm{F}=4.382$ and $96.66,10$ and $30 \mu \mathrm{M}$, respectively, $p<0.05)$. When compared, the mean distance moved in response to the first tone in the $30 \mu \mathrm{M}$ memantine-treated group was 2.5 times (Supplementary Figure $3 \mathrm{C} p<0.001$ ) 

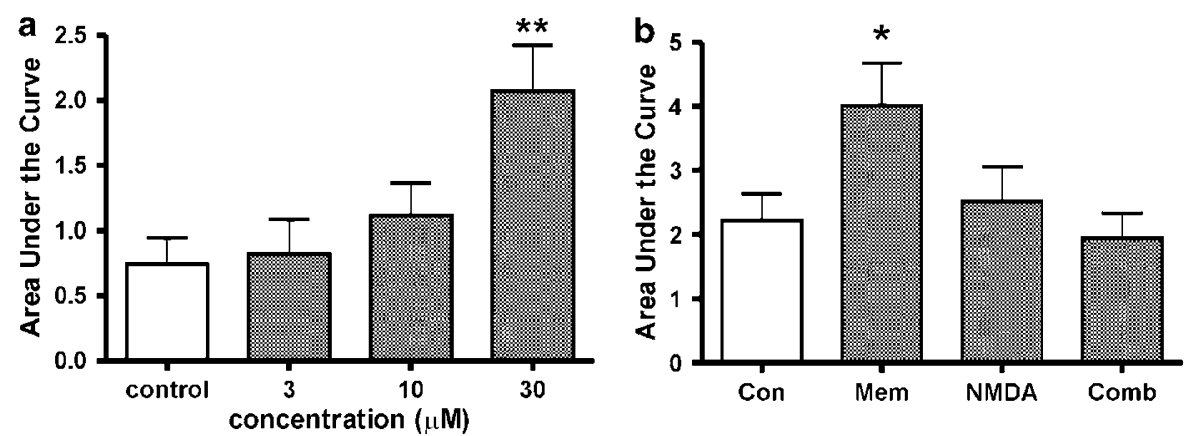

Figure 7 Memantine enhances startle response in zebrafish and is attenuated by NMDA. (a) Bar graph of AUC for control fish (clear) vs concentration response of memantine (3, I0, and $30 \mu \mathrm{M}$; shaded). Bars represent the average AUC $\pm S E M ; n=12$ per group. $* *$ $p<0.00$ I vs control group. (b) Bar graph of AUC of control (Con) fish (clear) vs memantine (Mem), NMDA, and combination (Comb) (shaded). Bars represent the average AUC \pm SEM; $n=10-12$ per group. $* p<0.05$ vs control group.

greater than noted in the control group. The distances moved in the $30 \mu \mathrm{M}$ memantine group also remained elevated for consecutive tones. Interestingly, the distances moved from the 10th tone onwards were increased compared to the control, although the ASR was only significant at the 15 th tone compared to the control group (Supplementary Figure 3C, $p<0.05$ ). To determine if this elevation was contributing to an increased effect on the overall distance moved, the AUC was calculated (Figure 7a). The AUC from all 50 tones demonstrated an effect of treatment (Figure 7a; one-way ANOVA, $\mathrm{F}=5.024$, $p<0.0001$ ), whereas the AUC from just the first 10 tones did not (data not shown), suggesting the elevation was having a significant contribution to the increased movement. Furthermore, it confirmed that the greatest effect was observed with $30 \mu \mathrm{M}$ (Figure 7a, post hoc Dunnett's $t$-test, $p<0.001)$, and hence this concentration was used for further experiments.

To confirm this response was mediated by the NMDA receptor, memantine was tested in combination with $30 \mu \mathrm{M}$ NMDA (Figure 7b, Supplementary Figure 4). Alone $30 \mu \mathrm{M}$ NMDA did not affect the habituation of the startle response compared to the control group (Figure $7 \mathrm{~b}$ NMDA; Supplementary Figure 4A), but when combined with memantine, it reduced the potentiation seen with memantine alone (Figure 7b Mem; Supplementary Figure 4B, $\mathrm{F}=25.87, p<0.0001$ ) to control levels (Figure 7b Comb; Supplementary Figure 4C).

\section{DISCUSSION}

A number of criteria have been proposed to determine whether a system is truly habituating or not (Thompson and Spencer, 1966; Brown, 1998). These were initially tested in the ASR of the zebrafish to determine if the iterative reduction seen in response to repeated acoustic stimuli was due to habituation. Previous studies investigating the hearing system of zebrafish of the $A B$ strain demonstrated that they were able to respond behaviorally to an acoustic stimulus after 5 d.p.f. from frequencies of $200 \mathrm{~Hz}$ upwards (Bang et al, 2002; Zeddies and Fay, 2005). We determined that in this particular set of experiments that $113 \mathrm{~dB}$ of $200 \mathrm{~Hz}$ gave the optimum measurable response. It is possible that the larvae's response to the sound stimulus was as a result of proprioceptive as well as auditory responses and so for the purposes of this discussion when referring to ASR both mechanisms are assumed to take place. The current study investigated whether this behavior could be further quantified to determine whether zebrafish were able to habituate to sound and which pathways modulate this effect.

Initially, we compared two strains of commonly used zebrafish to determine if there was any difference in the response to sound given via a loudspeaker. A number of different parameters such as number of fish, turn angle, acceleration, and velocity were assessed (data not shown) along with distance moved, and it was observed that the distance gave the best quantitative readout. Using the distance moved parameter, it was demonstrated that the WIK strain had an increased response to the sound over the AB strain. Further tests on the age of WIK strain fish suggested that distance moved was a strong correlate of the startle response and that 7 d.p.f. WIK fish were a reliable choice to use for assessment of ASR.

Having demonstrated that the startle response did decrease with subsequent tones, the objective was to determine whether this reduction fulfilled some of the criteria for habituation. The system showed that the zebrafish were able to recover their responsiveness to audio stimulation, an interval of $15 \mathrm{~min}$ in the same group of fish being sufficient to regain the startle responses to a second set of tones. Interestingly, there appeared to be an enhanced response to the second group of tones, suggesting a potentiation effect, possibly resulting from increased alertness (Kandel and Spencer, 1968; Groves and Thompson, 1970; Brown, 1998). However, more detailed work would be required to fully verify this enhanced effect. Initially, $1 \mathrm{~s}$ ITIs were used but when longer intervals were evaluated there was a different response; the longer ITIs leading to a slower habituation than that observed for the short ITIs. This is in keeping with the criterion that the more rapid the frequency of stimulation the more rapidly habituation is seen (Thompson and Spencer, 1966). In addition, we were then able to use the different ITIs to determine the effect of dishabituation. Dishabituation occurs when an entirely different type of stimulus reinitiates the startle response after habituation has occurred (Thompson and Spencer, 1966). In this particular case, a light pulse was used, as the change from light to dark possibly mimics 
the shadow of a predator causing a startle response (Easter and Nicola, 1996). Using the $5 \mathrm{~s}$ ITI, a $2 \mathrm{~s}$ pulse of light was interspersed between auditory tones without affecting the ITI. Reinduction of the startle response to the tones did indeed occur in those larvae exposed to the light pulse. Thus, the behavior displayed by the zebrafish to the sequential auditory cues was consistent with the phenomenon of habituation demonstrating that the iterative reductions seen were not merely a result of simple motor exhaustion.

Previous studies have linked rolipram with an increase in learning and memory or reversal of pharmacological impairment of memory in rodents (Imanishi et al, 1997; Barad et al, 1998; Zhang et al, 2000; Zhang and O'Donnell, 2000). Interestingly, when administered to rats, rolipram demonstrated an increase in the mean amplitude of the ASR, which was primarily mediated by the (-)-isomer of the compound (Kehne et al, 1991). In the current assay, an increase in the distance moved was seen in fish exposed to rolipram for $24 \mathrm{~h}$ (particularly at $3 \mu \mathrm{M}$ ). These findings are consistent with an enhanced startle response and appear similar to the effects reported for rolipram in the rat. These results with rolipram may indicate the presence of phosphodiesterase type IV enzyme in the zebrafish brain, or at least high-affinity rolipram binding sites. However, experiments using selective, non-rolipram-based PDE IV inhibitors would be required to confirm the presence of PDE IV in zebrafish and that the effects of rolipram were in fact due to true inhibition of this enzyme.

In order to determine whether a potentiating effect on the ASR (and a delayed habituation response) could also be achieved with other agents linked to memory enhancing effects, donepezil and memantine were evaluated in the assay since they act through different pharmacological mechanisms, donepezil being an acetylcholinesterase inhibitor (Sugimoto et al, 2002) and memantine an NMDA receptor antagonist (Parsons et al, 1999). Both are current therapies for Alzheimer's disease and have been shown to improve cognitive ability in Alzheimer's disease patients (Burns et al, 1999; Winblad et al, 2001, 2006; Peskind et al, 2006; Takeda et al, 2006). In the present study, both of these compounds also increased the distance moved in response to the acoustic stimulus in a similar manner to rolipram.

The effect of memantine in this model is consistent with an effect at NMDA receptors since coadministration with the agonist NMDA abrogated the ASR potentiating effects seen with memantine alone. The data also further confirm a functional effect of NMDA receptors in zebrafish. It is of interest to note that the profile (time course) of memantine on the ASR habituation paradigm differed from those observed with rolipram and donepezil in zebrafish; memantine producing a smaller, more prolonged effect compared to the immediate, relatively short lasting potentiation of ASR noted with rolipram and donepezil. It has been reported that the NMDA antagonist dizocilpine (MK-801) induces amnesia in goldfish using active-avoidance conditioning as the learning regimen (Xu et al, 1998). However, in contrast to the amnestic effects of dizocilpine in goldfish, dizocilpine did not impair retention of avoidance responses in zebrafish trained to swim from a lighted compartment to a dark compartment to avoid an electrical shock to the body (Xu et al, 2007). In the current study, an effect was seen with memantine decreasing the habituation to a single repeated stimulus and it is impossible to conclude whether this effect was due to the NMDA antagonist causing the zebrafish to forget that another stimulus is about to follow or whether it simply increased alertness of the fish. Interestingly, a previous study investigating the effect of the NMDA antagonist, dizocilpine, on the habituation of the ASR in mice demonstrated an enhanced startle effect compared to controls (Klamer et al, 2004), which is similar to the findings with memantine of this study using zebrafish.

In mammals, expression of five NMDA receptor genes has been reported; one encoding an NMDAR1 subunit and four encoding four NMDAR2 subunits (Ishii et al, 1993). Cox et al (2005) reported that the genes for the five receptor subunits exist in zebrafish, with each consisting of two paralogous genes. In rats, the NMDAR1 subunit is distributed ubiquitously in the brain, whereas the NMDAR2 subunits exhibit distinct regional patterns in the brain. In zebrafish, the two NMDAR1 genes were found in the brain at 48 hours post fertilization, whereas only one of the NMDAR2D paralogue genes was found in the brain at this time (Cox et al, 2005). It is likely that the effects of memantine in zebrafish larvae are mediated via the NMDAR1, although only selective agonists and antagonists at the various subunits will substantiate the exact subtype responsible.

A number of studies in zebrafish and mammals have demonstrated learning effect through the nicotinic (Levin and Simon, 1998; Power et al, 2003; Levin et al, 2006a) and muscarinic (Levin and Simon, 1998; Power et al, 2003; Levin et al, 2006a) cholinergic receptors. Levin and Chen (2004) and Levin et al (2006b) have studied extensively the effects of nicotine on learning and memory in zebrafish and have shown that low doses of nicotine enhance memory while higher doses impair memory in zebrafish. Zebrafish larvae possess acetylcholinesterase with similar enzymatic activity to other vertebrates, but do not have butyrylcholinesterase (Svoboda et al, 2002), and cloning and binding experiments have demonstrated the presence of nicotinic ( $\beta 3, \alpha 2$, and $\alpha 7$ subunits) (Zirger et al, 2003) and muscarinic receptors (Williams and Messer, 2004) in zebrafish embryo. To determine whether the enhanced startle response observed with the cholinesterase inhibitor donepezil was mediated via either nicotinic or muscarinic receptors or both types of receptors, donepezil treatment was combined with the nicotinic antagonist mecamylamine or the muscarinic antagonist atropine. Interestingly, the effect of donepezil appeared to be mediated primarily via nicotinic receptors. Mecamylamine abolished completely the donepezil-induced enhancement of ASR and (donepezil-induced) decrease in habituation, whereas atropine failed to influence the effects of donepezil when given together. This was despite atropine having an inhibitory effect in its own right on the ASR. In fact, it appeared that donepezil caused a greater effect in the presence of atropine than when given alone. At present, the mechanism(s) by which atropine appears to potentiate the effects of donepezil is unclear; effects at pre- and postjunctional muscarinic receptors could be explored with the use of selective antagonists. Overall, the results obtained with donepezil in zebrafish larvae support an effect on 
habituation behavior via nicotinic receptors, which is in keeping with previous findings demonstrating a cognitive enhancing effect of nicotine in adult zebrafish (Levin et al, 2006a).

Owing to the large requirement for compound and numbers in mammalian models, alternative behavioral models are increasingly being sought. These models need to be a reliable and predictable reflection of rodent and ultimately human behavior. Furthermore, a model that is amenable to high-throughput screening, this makes them more attractive for use. These criteria become particularly demanding when it comes to modelling aspects of cognition. A number of recent studies have demonstrated the use of zebrafish as a valid subject for pharmacological modelling of behaviors and disorders seen in mammals (for review see Goldsmith, 2004), with a number of procedures using adult zebrafish having been developed for learning and memory (Williams et al, 2002; Carvan et al, 2004; Swain et al, 2004; Levin et al, 2006a). These have adapted rodent tests such as the T-maze and place preference for an aquatic environment. Despite the fact that these tests are able to reproduce behaviors and changes to pharmacological challenges akin to the rodent, the use of adults prevents these tests being used as truly high-throughput screens. Adult zebrafish are around $2.5 \mathrm{~cm}$ in length and are typically live in volumes of 1-21 per fish. In contrast, larval zebrafish are only a few millimeters in length and can be kept in 96-well plate format.

The current study set out to determine if zebrafish larvae could be used to study learning. To our knowledge, there have been no published studies investigating the habituation of the ASR in larval zebrafish. We demonstrated that larvae not only display behavior consistent with habituation but that they can be modulated pharmacologically in similar ways to the rodent. Since all the compounds tested here have been linked to improved learning and memory, these results suggest that the enhancing effect on ASR (decreased habituation) may reflect an increase in alertness or awareness in the fish. However, other investigations have interpreted the enhancement of the response as a deficit of habituation reflecting cognitive impairments, since schizophrenic patients show enhancement of their ASR (Klamer et al, 2004; Meincke et al, 2004); therefore, more investigations clearly need to be conducted into to this area of cognition.

In summary, we have developed an assay in zebrafish amenable to high-throughput screening, which resembles the ASR of rodents. Experiments are ongoing to establish whether there are interactive/cooperative links between biochemical/receptor pathways activated by the pharmacological agents used in this study. However, this assay has been shown to identify drugs which currently play a role in enhancing learning and memory, and can be developed further to be used as a screen to identify compounds for further study in cognition.

\section{ACKNOWLEDGEMENTS}

Part of this work was funded by an SBRI Grant from the BBSRC BBS/B/14906. We would like to thank the DanioLabs Ltd aquarium staff for their care and maintenance of the fish facility.

\section{DISCLOSURE/CONFLICT OF INTEREST}

The authors are employees of DanioLabs Ltd (a wholly owned subsidiary of VASTox plc) and are stock and/or options holders in VASTox plc.

\section{REFERENCES}

Bang PI, Yelick PC, Malicki JJ, Sewell WF (2002). High-throughput behavioral screening method for detecting auditory response defects in zebrafish. J Neurosci Methods 118: 177-187.

Barad M, Bourtchouladze R, Winder DG, Golan H, Kandel E (1998). Rolipram, a type IV-specific phosphodiesterase inhibitor, facilitates the establishment of long-lasting long-term potentiation and improves memory. Proc Natl Acad Sci USA 95: $15020-15025$.

Brown GD (1998). Nonassociative learning processes affecting swimming probability in the seaslug Tritonia diomedea: habituation, sensitization and inhibition. Behav Brain Res 95: 151-165.

Burns A, Rossor M, Hecker J, Gauthier S, Petit H, Moller HJ et al (1999). The effects of donepezil in Alzheimer's disease - results from a multinational trial. Dement Geriatr $\operatorname{Cogn}$ Disord 10: 237-244.

Cahill GM (2002). Clock mechanisms in zebrafish. Cell Tissue Res 309: 27-34.

Carvan III MJ, Loucks E, Weber DN, Williams FE (2004). Ethanol effects on the developing zebrafish: neurobehavior and skeletal morphogenesis. Neurotoxicol Teratol 26: 757-768.

Cho W, Heberlein U, Wolf FW (2004). Habituation of an odorantinduced startle response in Drosophila. Genes Brain Behav 3: 127-137.

Cox JA, Kucenas S, Voigt MM (2005). Molecular characterization and embryonic expression of the family of $N$-methylD-aspartate receptor subunit genes in the zebrafish. Dev Dyn 234: 756-766.

Easter Jr SS, Nicola GN (1996). The development of vision in the zebrafish (Danio rerio). Dev Biol 180: 646-663.

Goldsmith P (2004). Zebrafish as a pharmacological tool: the how, why and when. Curr Opin Pharmacol 4: 504-512.

Groves PM, Thompson RF (1970). Habituation: a dual-process theory. Psychol Rev 77: 419-450.

Grunwald DJ, Eisen JS (2002). Headwaters of the zebrafishemergence of a new model vertebrate. Nat Rev Genet 3: 717-724.

Guo S (2004). Linking genes to brain, behavior and neurological diseases: what can we learn from zebrafish? Genes Brain Behav 3: 63-74.

Haffter P, Granato M, Brand M, Mullins MC, Hammerschmidt M, Kane DA et al (1996). The identification of genes with unique and essential functions in the development of the zebrafish, Danio rerio. Development 123: 1-36.

Imanishi T, Sawa A, Ichimaru Y, Miyashiro M, Kato S, Yamamoto $\mathrm{T}$ et al (1997). Ameliorating effects of rolipram on experimentally induced impairments of learning and memory in rodents. Eur J Pharmacol 321: 273-278.

Ishii T, Moriyoshi K, Sugihara H, Sakurada K, Kadotani H, Yokoi M et al (1993). Molecular characterization of the family of the $N$-methyl-D-aspartate receptor subunits. J Biol Chem 268: 2836-2843.

Kandel ER, Spencer WA (1968). Cellular neurophysiological approaches in the study of learning. Physiol Rev 48: 65-134.

Kehne JH, Boulis NM, Davis M (1991). Effects of the phosphodiesterase inhibitor rolipram on the acoustic startle response in rats. Psychopharmacology (Berlin) 105: 27-36.

Kimmel CB, Ballard WW, Kimmel SR, Ullmann B, Schilling TF (1995). Stages of embryonic development of the zebrafish. Dev Dyn 203: 253-310. 
Klamer D, Palsson E, Revesz A, Engel JA, Svensson L (2004). Habituation of acoustic startle is disrupted by psychotomimetic drugs: differential dependence on dopaminergic and nitric oxide modulatory mechanisms. Psychopharmacology (Berlin) 176: $440-450$.

Koch M (1999). The neurobiology of startle. Prog Neurobiol 59: 107-128.

Levin ED, Chen E (2004). Nicotinic involvement in memory function in zebrafish. Neurotoxicol Teratol 26: 731-735.

Levin ED, Limpuangthip J, Rachakonda T, Peterson M (2006a). Timing of nicotine effects on learning in zebrafish. Psychopharmacology (Berlin) 184: 547-552.

Levin ED, McClernon FJ, Rezvani AH (2006b). Nicotinic effects on cognitive function: behavioral characterization, pharmacological specification, and anatomic localization. Psychopharmacology (Berlin) 184: 523-539.

Levin ED, Simon BB (1998). Nicotinic acetylcholine involvement in cognitive function in animals. Psychopharmacology (Berlin) 138: 217-230.

Luque MA, Perez-Perez MP, Herrero L, Torres B (2005). Involvement of the optic tectum and mesencephalic reticular formation in the generation of saccadic eye movements in goldfish. Brain Res Rev 49: 388-397.

Marcus EA, Nolen TG, Rankin CH, Carew TJ (1988). Behavioral dissociation of dishabituation, sensitization, and inhibition in Aplysia. Science 241: 210-213.

Meincke U, Light GA, Geyer MA, Braff DL, Gouzoulis-Mayfrank E (2004). Sensitization and habituation of the acoustic startle reflex in patients with schizophrenia. Psychiatry Res 126: $51-61$.

Ninkovic J, Folchert A, Makhankov YV, Neuhauss SC, Sillaber I, Straehle U et al (2006). Genetic identification of AChE as a positive modulator of addiction to the psychostimulant D-amphetamine in zebrafish. J Neurobiol 66: 463-475.

Orger MB, Gahtan E, Muto A, Page-McCaw P, Smear MC, Baier H (2004). Behavioral screening assays in zebrafish. Methods Cell Biol 77: 53-68.

Parsons CG, Danysz W, Quack G (1999). Memantine is a clinically well tolerated $\mathrm{N}$-methyl-D-aspartate (NMDA) receptor antagonist-a review of preclinical data. Neuropharmacology 38: 735-767.

Peskind ER, Potkin SG, Pomara N, Ott BR, Graham SM, Olin JT et al (2006). Memantine treatment in mild to moderate Alzheimer disease: a 24-week randomized, controlled trial. Am J Geriatr Psychiatry 14: 704-715.

Pletnikov MV, Storozheva ZI, Sherstnev VV (1995). Developmental analysis of habituation of acoustic startle in the preweanling and adult rats. Behav Processes 34: 269-278.

Postlethwait JH, Woods IG, Ngo-Hazelett P, Yan YL, Kelly PD, Chu $\mathrm{F}$ et al (2000). Zebrafish comparative genomics and the origins of vertebrate chromosomes. Genome Res 10: 1890-1902.

Power AE, Vazdarjanova A, McGaugh JL (2003). Muscarinic cholinergic influences in memory consolidation. Neurobiol Learn Mem 80: 178-193.

Sugimoto H, Ogura H, Arai Y, Limura Y, Yamanishi Y (2002). Research and development of donepezil hydrochloride, a new type of acetylcholinesterase inhibitor. Jpn J Pharmacol 89: $7-20$

Svoboda KR, Vijayaraghavan S, Tanguay RL (2002). Nicotinic receptors mediate changes in spinal motoneuron development and axonal pathfinding in embryonic zebrafish exposed to nicotine. J Neurosci 22: 10731-10741.
Swain HA, Sigstad C, Scalzo FM (2004). Effects of dizocilpine (MK-801) on circling behavior, swimming activity, and place preference in zebrafish (Danio rerio). Neurotoxicol Teratol 26: 725-729.

Takeda A, Loveman E, Clegg A, Kirby J, Picot J, Payne E et al (2006). A systematic review of the clinical effectiveness of donepezil, rivastigmine and galantamine on cognition, quality of life and adverse events in Alzheimer's disease. Int J Geriatr Psychiatry 21: 17-28.

Thompson RF, Spencer WA (1966). Habituation: a model phenomenon for the study of neuronal substrates of behavior. Psychol Rev 73: 16-43.

Tropepe V, Sive HL (2003). Can zebrafish be used as a model to study the neurodevelopmental causes of autism? Genes Brain Behav 2: 268-281.

Westerfield M (2000). The Zebrafish Book. A Guide for the Laboratory Use of Zebrafish (Danio rerio). University of Oregon Press: Eugene, OR.

Whitfield TT (2002). Zebrafish as a model for hearing and deafness. J Neurobiol 53: 157-171.

Williams FE, Messer Jr WS (2004). Muscarinic acetylcholine receptors in the brain of the zebrafish (Danio rerio) measured by radioligand binding techniques. Comp Biochem Physiol $C$ Toxicol Pharmacol 137: 349-353.

Williams FE, White D, Messer WS (2002). A simple spatial alternation task for assessing memory function in zebrafish. Behav Processes 58: 125-132.

Winblad B, Engedal K, Soininen H, Verhey F, Waldemar G, Wimo A et al (2001). A 1-year, randomized, placebo-controlled study of donepezil in patients with mild to moderate AD. Neurology 57: 489-495.

Winblad B, Kilander L, Eriksson S, Minthon L, Batsman S, Wetterholm AL et al (2006). Donepezil in patients with severe Alzheimer's disease: double-blind, parallel-group, placebocontrolled study. Lancet 367: 1057-1065.

Wullimann MF, Mueller T (2004). Teleostean and mammalian forebrains contrasted: evidence from genes to behavior. J Comp Neurol 475: 143-162.

Xu X, Boshoven W, Lombardo B, Spranger J (1998). Comparison of the amnestic effects of NMDA receptor antagonist MK-801 and nitric oxide synthase inhibitors: L-NAME and L-NOARG in goldfish. Behav Neurosci 112: 892-899.

Xu X, Scott-Scheiern T, Kempker L, Simons K (2007). Active avoidance conditioning in zebrafish (Danio rerio). Neurobiol Learn Mem 87: 72-77.

Zeddies DG, Fay RR (2005). Development of the acoustically evoked behavioral response in zebrafish to pure tones. J Exp Biol 208: 1363-1372.

Zhang HT, Crissman AM, Dorairaj NR, Chandler LJ, O'Donnell JM (2000). Inhibition of cyclic AMP phosphodiesterase (PDE4) reverses memory deficits associated with NMDA receptor antagonism. Neuropsychopharmacology 23: 198-204.

Zhang HT, O'Donnell JM (2000). Effects of rolipram on scopolamine-induced impairment of working and reference memory in the radial-arm maze tests in rats. Psychopharmacology (Berlin) 150: 311-316.

Zhdanova IV, Wang SY, Leclair OU, Danilova NP (2001). Melatonin promotes sleep-like state in zebrafish. Brain Res 903: 263-268.

Zirger JM, Beattie CE, McKay DB, Boyd RT (2003). Cloning and expression of zebrafish neuronal nicotinic acetylcholine receptors. Gene Expr Patterns 3: 747-754.

Supplementary Information accompanies the paper on the Neuropsychopharmacology website (http://www.nature.com/npp) 